\title{
Nominal PbSe nano-islands on PbTe: grown by MBE, analyzed by AFM and TEM
}

\author{
Peter Moeck ${ }^{1}$, Mukes Kapilashrami ${ }^{1 *}$, Jeahuck Lee ${ }^{2}$, James E. Morris ${ }^{2}$, Nigel D. Browning ${ }^{3}$, and Patrick J. McCann ${ }^{4}$ \\ ${ }^{1}$ Portland State University, Department of Physics, P.O. Box 751, Portland, OR 97207-0751, (pmoeck@pdx.edu), * Department of \\ Materials Science, The Royal Institute of Technology, Brinellvägen 23, S-100 44 Stockholm, Sweden \\ ${ }^{2}$ Department of Electrical \& Computer Engineering, P.O. Box 751, Portland, OR 97207-0751 \\ ${ }^{3}$ Department of Chemical Engineering and Materials Science, University of California at Davis, One Shields Avenue, Davis, CA 95616; and \\ National Center for Electron Microscopy, MS 72-150, Lawrence Berkeley National Laboratory, Berkeley, CA 94720 \\ ${ }^{4}$ University of Oklahoma, School of Electrical and Computer Engineering, Norman, OK 73019
}

\begin{abstract}
Nominal PbSe nano-islands were grown in the Stranski-Krastanow mode on (111) oriented $\mathrm{PbTe} / \mathrm{BaF}_{2}$ pseudo-substrates by molecular beam epitaxy (MBE). The morphology and number density of these islands were assessed by means of atomic force microscopy (AFM). Transmission electron microscopy (TEM) was employed to determine the strain state and crystallographic structure of these islands. On the basis of both AFM and TEM analyses, we distinguish between different groups of islands.
\end{abstract}

Keywords: AFM, TEM. MBE, PbSe and nanoislands

\section{Introduction}

Heteroepitaxial semiconductor quantum dots are expected to lead to "paradigm changes in semiconductor physics" [1]. As reviewed in recent textbooks [2,3], improved optoelectronic devices (such as lasers and mid-infrared detectors) and novel nanoelectronics concepts which depend on single electron transport and tunneling have either been realized or may be demonstrated as the field matures.

The $\mathrm{PbSe} / \mathrm{PbTe}$ quantum dot system is particularly interesting for mid-infrared vertical cavity surface emitting lasers [4]. Unlike most III-V and II-VI compound semiconductor quantum dot systems, strong confinement of both holes and electrons can readily be achieved in lead salt quantum dots. The Bohr radii of the holes and electrons are of the same order of magnitude, resulting for $\mathrm{PbSe}$ in an exciton Bohr radius on the order of magnitude $50 \mathrm{~nm}$.

Materials science aspects of heteroepitaxial quantum dot growth are extensively discussed in the above mentioned textbooks, but atomic ordering and phase separation in such heteroepitaxial structures are a relative new field of study [5,6]. This is rather surprising, as it has been known since 1985 that atomic ordering and phase separation exists in basically all technically important heteroepitaxial column IV element, III-V, and II-VI compound semiconductor alloy structures that possess in the disordered state the diamond or sphalerite (zinc blende) structure [7-9].

Atomic ordering and phase separation in IV-VI compounds with the halite (rock salt) structure has, to the best of our knowledge, only been reported twice in epitaxial $(\mathrm{Pb}, \mathrm{Eu}) \mathrm{Te}$ [10,11]. Preliminary TEM results showed that there is also atomic ordering somewhere in the structure of free-standing three-dimensional (3D) nominal $\mathrm{PbSe}$ nano-islands on nominal $\mathrm{PbSe}$ wetting layers that were grown in the Stranski-Krastanow mode by means of MBE on $\mathrm{PbTe} / \mathrm{BaF}_{2}$ pseudo-substrates [12].

Note that while typical heteroepitaxial growth of quantum dots proceeds in the Stranski-Krastanow growth mode under compressive stress, there is a tensile stress on nominal $\mathrm{PbSe}$ islands and wetting layers on a $\mathrm{PbTe} / \mathrm{BaF}_{2}$ pseudo-substrate. This results in a tensile strain of a few percent for nominal $\mathrm{PbSe}$ quantum dots. As in the better studied (compressively strained) III-V quantum dot systems, e.g. [13] and references therein, there is most likely also alloying in both the wetting layer and the nanoislands. This alloying will tend to reduce the nominal stresses and strains.

For simplicity, we will consider both the 3D islands and the wetting layer as being composed of $\mathrm{Pb}(\mathrm{Se}, \mathrm{Te})$. As in the case of epitaxial $(\mathrm{Pb}, \mathrm{Eu}) \mathrm{Te}$ $[10,11]$, the nominal structure of $\mathrm{Pb}(\mathrm{Se}, \mathrm{Te})$ would be that of the uppermost substrate layer, i.e. $\mathrm{PbTe}$, which possessed the halite prototype structure. When such nano-islands are overgrown with a larger band gap semiconductor material (with a larger lattice constant) such as PbTe, (tensibly) strained quantum dots are obtained. We will, therefore, refer to the $\mathrm{Pb}(\mathrm{Se}, \mathrm{Te})$ nano-islands on $\mathrm{Pb}(\mathrm{Se}, \mathrm{Te})$ wetting layer we investigated here below as predecessor structures of quantum dots.

In this paper we report preliminary results of an atomic force microscopy study of such a sample. Transmission electron microscopy (TEM) results from both the nano-island and the wetting layer of the same sample will also be presented. 


\section{Experimental Details}

The nominal $\mathrm{PbSe}$ islands on $\mathrm{PbTe}$ were grown by means of MBE at the University of Oklahoma using $\mathrm{PbTe}, \mathrm{PbSe}$ and $\mathrm{Se}$ sources. Freshly cleaved (111) oriented $\mathrm{BaF}_{2}$ was used as the substrate. After desorption of surface layers for 15 minutes at $500{ }^{\circ} \mathrm{C}$, a $2.67 \mu \mathrm{m}$ thick, lattice matched $\mathrm{PbTe}_{0.22} \mathrm{Se}_{0.78}$ buffer layer was grown at $400{ }^{\circ} \mathrm{C}$ and a growth rate of 0.278 $\mathrm{nm} \mathrm{s}^{-1}$. At the same temperature, $1.8 \mu \mathrm{m}$ Se doped $\mathrm{PbTe}$ was grown at a rate of $0.167 \mathrm{~nm} \mathrm{~s}^{-1}$. Then the temperature was lowered to $380{ }^{\circ} \mathrm{C}$ and $0.8 \mathrm{~nm}(2.6$ ML) $\mathrm{PbSe}$ was deposited at a rate of $0.027 \mathrm{~nm} \mathrm{~s}^{-1}$. The sample was finally cooled down to room temperature at a rate of $1 \mathrm{~K} \mathrm{~s}^{-1}$.

The AFM study was performed at Portland State University using the non-contact mode of a Questant Q-Scope ${ }^{\mathrm{TM}} 250$. The radius of the scanning Si tip on the AFM cantilever was nominally less than $10 \mathrm{~nm}$.

From this $\mathrm{Pb}(\mathrm{Se}, \mathrm{Te})$ quantum dot predecessor structure, [111] plan-view TEM specimens were prepared by standard mechanical grinding and ionmilling techniques. These specimens were analyzed in a JEOL JEM-3010 TEM at $300 \mathrm{kV}$ at the Research Resources Center of the University of Illinois at Chicago.

\section{Results and Discussion}

\section{AFM assessment}

Figs. 1, 2, and 3a-c show typical three- and twodimensional (2D) AFM images of the $\mathrm{Pb}(\mathrm{Se}, \mathrm{Te})$ quantum dot predecessor structure. As the island height (horizontal) sensitivity of an AFM is typically much higher than the island width (vertical) sensitivity, the horizontal steps that are resolved in Figs. 1 and 2, are quite interesting and warrant further investigation. Perhaps these steps might be due to atomic terraces, as observed earlier by AFM on III-V compound semiconductor nano-islands [14].

Fig. 3a-c show different populations of nanoisland. Note the increasing height scale from maximal $62.74 \mathrm{~nm}$ in the top image, Fig. 3a, over $101.8 \mathrm{~nm}$ in the middle image, Fig. $3 \mathrm{~b}$, to maximal $163.4 \mathrm{~nm}$ in the bottom image, Fig. 3c. Obviously regions with smaller and lager islands coexist, Fig. 3a and $3 \mathrm{~b}$, and occasionally there are a few very large islands, Fig. 3c. Only the largest islands, e.g. Fig. 3c, seem to reveal facetted side walls. This indicates that there are significant convolution effects for smaller islands due to the usage of a rather blunt AFM tip. As it is well known, convolution effects between the shape of an AFM tip and the shape of nano-islands are unavoidable [15].

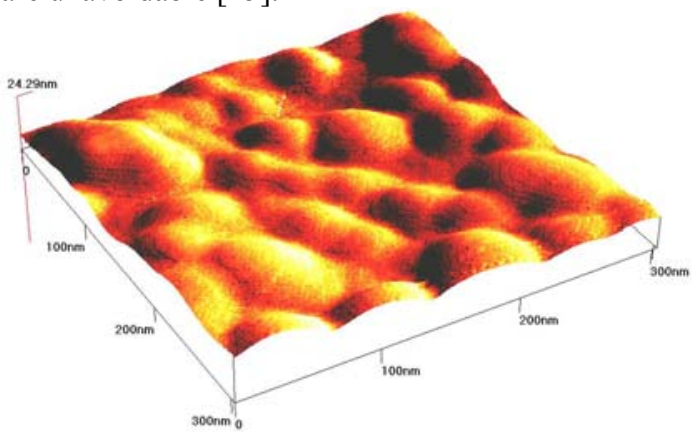

Figure 1. 3D-AFM image of an approximately 0.09 $\mu \mathrm{m}^{2}$ wide section of the $\mathrm{Pb}(\mathrm{Se}, \mathrm{Te})$ quantum dot predecessor structure. Note the faintly resolved terraces that we will investigate further.

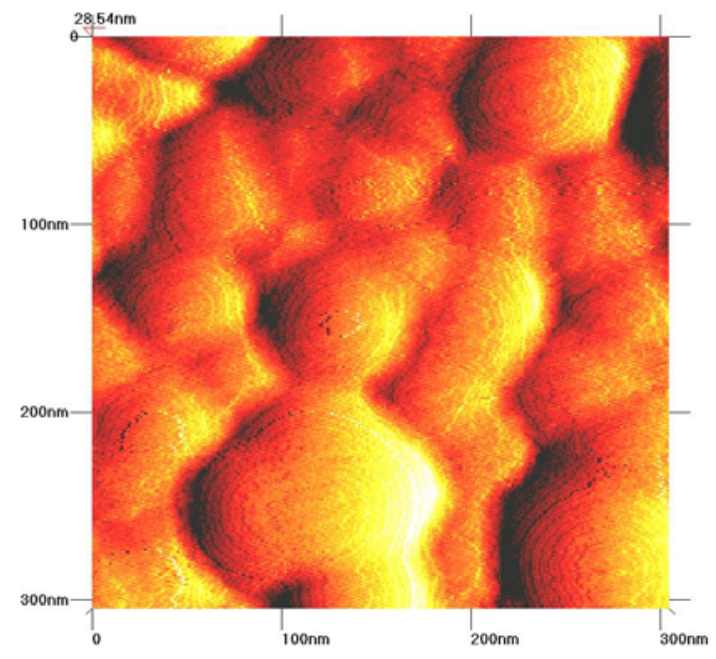

Figure 2. 2D-AFM image of an approximately 0.09 $\mu \mathrm{m}^{2}$ wide section of the $\mathrm{Pb}(\mathrm{Se}, \mathrm{Te})$ quantum dot predecessor structure. Note the well resolved terraces that we will investigate further.

Since the AFM tip size is constant, these convolutions are less severe for nano-islands that are much wider than the tip radius. The usage of a rather blunt AFM may be the reason why we did not observe the shape of the nano-islands as being that of trigonal pyramids with $<100>$ side facets, as other authors $[16,17]$ reported for very similar samples.

On the basis of AFM measurements we group the nano-islands into three groups according to well resolved peaks in the height histograms of 1583 assessed nano-islands. In total, an area of $31 \mu \mathrm{m}^{2}$ was assessed and the number density of nano-islands of all three groups adds up to approximately $51 \mu^{-2}$. 
For simplicity we refer to these groups as: small, medium height, and tall nano-islands. Small islands possess a number density of $4.7 \mu^{-2}$ and have an average height $(\mathrm{h})$ of $8.2 \mathrm{~nm}$ with a standard deviation $(\sigma)$ of $1.6 \mathrm{~nm}$, resulting in a relative spread $(\% / \mathrm{h})$ of $19.5 \%$. Medium-height islands are with a number density of $41 \mu^{-2}$ in the majority and possess an average height of $16.9 \mathrm{~nm}$ with a standard deviation of $4.6 \mathrm{~nm}$ and a relative spread of $27.2 \%$. Tall islands possess a number density of $5.4 \mu^{-2}$ and have an average height of $41.1 \mathrm{~nm}$, standard deviation of $10.9 \mathrm{~nm}$, and relative spread of $26.5 \%$.

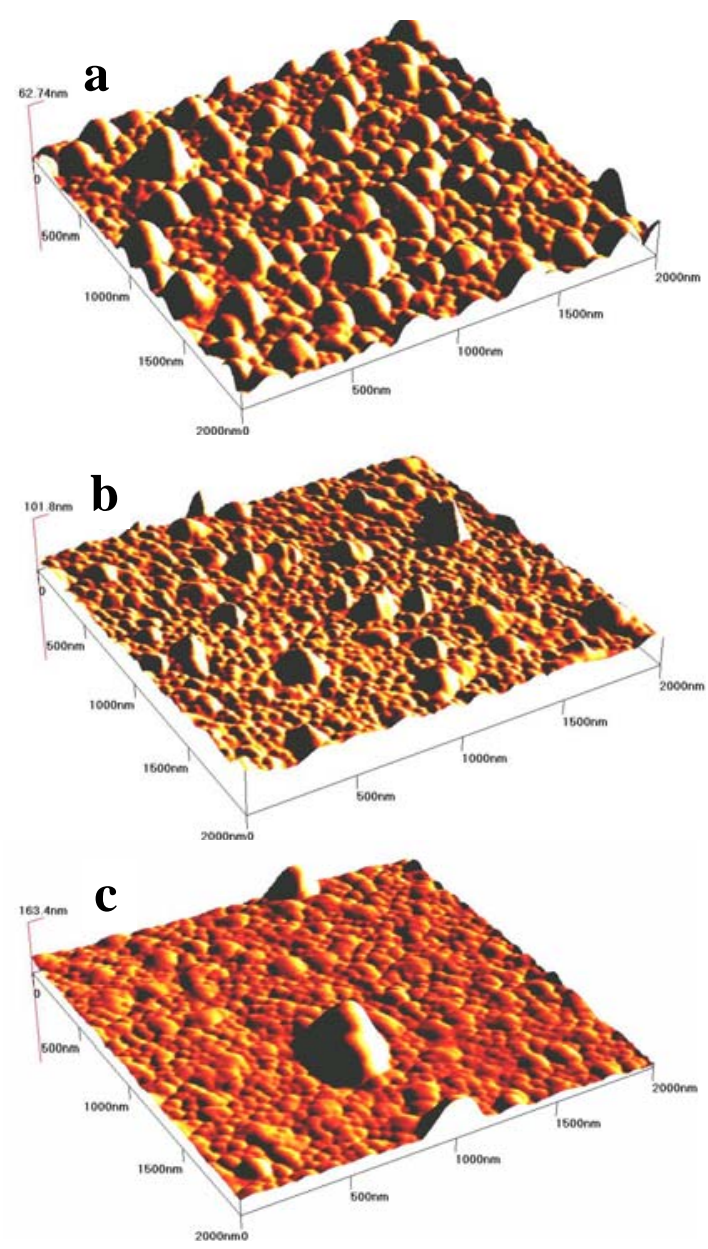

Figure 3. 3D-AFM images of $4 \mu \mathrm{m}^{2}$ wide sections of the $\mathrm{Pb}(\mathrm{Se}, \mathrm{Te})$ quantum dot predecessor structure. Note the increasing height scale in these images from top (a - maximal $62.74 \mathrm{~nm}$ ) to bottom (c - maximal $163.4 \mathrm{~nm}$ ). Obviously regions with smaller and lager islands coexist, (a) and (b) and occasionally there are a few very large islands (c).

\section{TEM study}

Fig. 4 shows smaller (revealed by so-called "coffee-bean contrasts" and larger (revealed by socalled "black-white" contrasts) $\mathrm{Pb}(\mathrm{Se}, \mathrm{Te})$ nanoislands. According to the well known Ashby-Brown theory of strained precipitates, the differences in contrast are due to differences in size. The presence of strained nano-island that can be classified into two different size (and height) groups is, thus confirmed by TEM. As the contrasts in this image show, these two kinds of islands are obviously strained and we call them ordinarily strained islands. They may, thus, be considered to constitute predecessors of ordinarily strained quantum dots. The widest (and tallest) nanoislands that were observed by means of AFM are probably relaxed and may, therefore, not act as quantum dots when overgrown with a larger bandgap semiconductor layer.

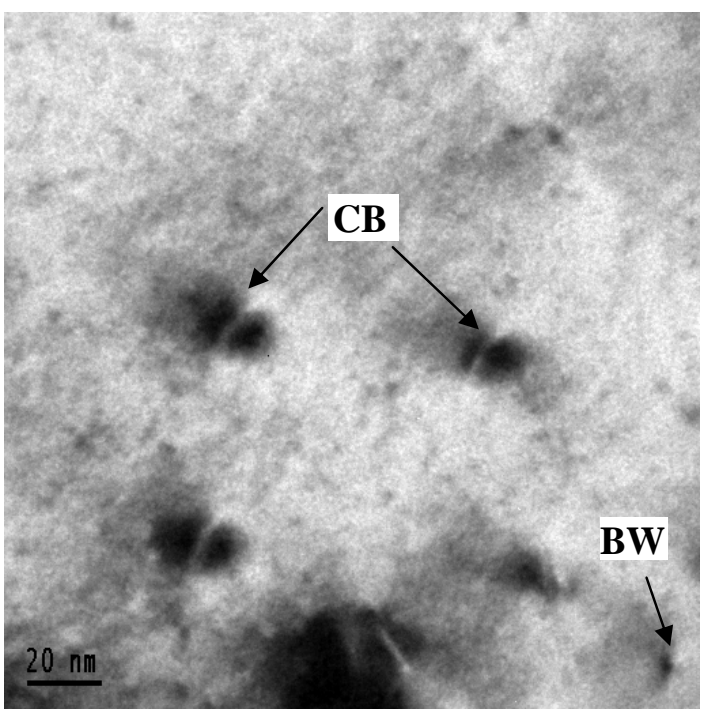

Figure 4. Near $<111\rangle,\{220\}$ dark field diffraction contrast image of smaller and larger ordinarily strained $\mathrm{Pb}(\mathrm{Se}, \mathrm{Te})$ quantum dot predecessor islands. Typical "black-white" contrasts (marker BW and arrow) and "coffee-bean" contrasts (marker CB and arrows) indicate that the nano-islands are fully strained and differ in size.

Fig. 5 shows a small region of the wetting layer between islands in high resolution in the [111] zone axis orientation. The three approximately $0.22 \mathrm{~nm}$ wide $\pm\{-220\}$ lattice spacings that are revealing of the [111] zone-axis pattern of a crystal with halite structure are clearly resolved in both the image and its insert Fourier transform power spectrum. 


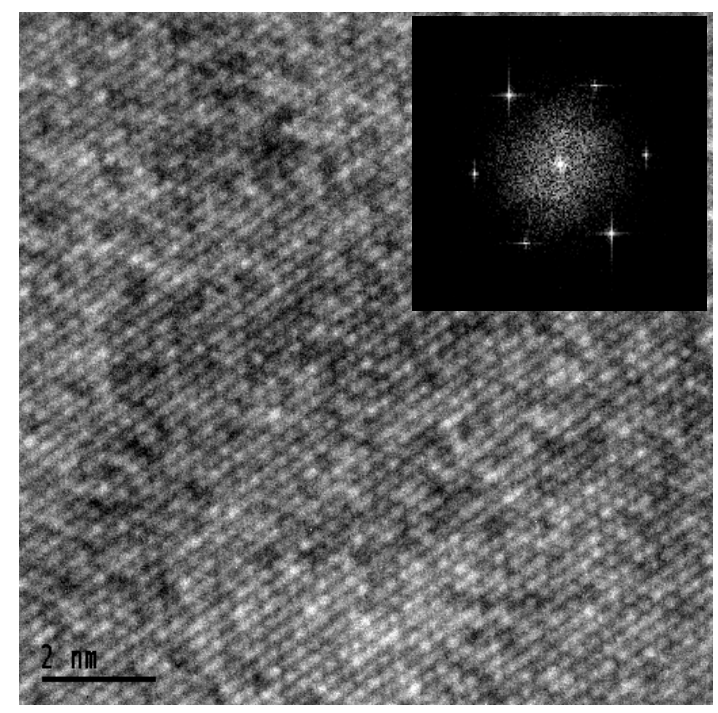

Figure 5. [111] zone axis, high-resolution phasecontrast image with insert Fourier transform power spectrum.

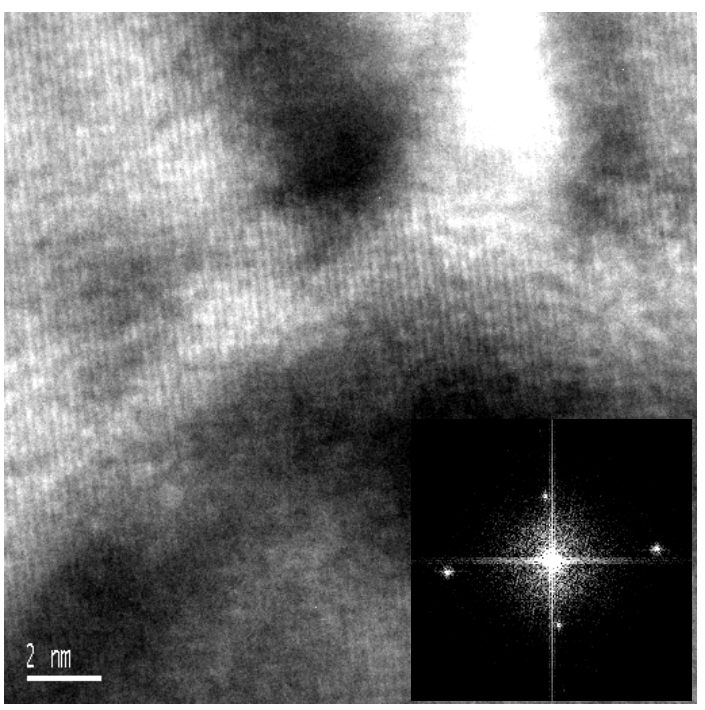

Figure 6. [112] zone axis, high-resolution phasecontrast image with insert Fourier transform power spectrum. "Pseudo black-white" contrast is visible in the image, indicating the presence of small strained $\mathrm{Pb}(\mathrm{Se}, \mathrm{Te})$ quantum dot predecessor islands.

Fig. 6 shows the sample in the [112] zone-axis orientation. The approximately $0.22 \mathrm{~nm}$ wide \pm (220) lattice spacing and the $0.36 \mathrm{~nm}$ wide $\pm(11-1)$ lattice spacing that are revealing of the [112] zone axis pattern of a crystal with halite structure are clearly resolved in both the image and its insert Fourier transform power spectrum.
Adjacent to the areas with ordinarily strained nano-islands, there were specimen regions that showed under the same imaging condition contrasts that are probably mainly due to absorption differences, Fig. 7. We, therefore, suggest that these entities possess significantly higher Te contents than the surrounding matrix.

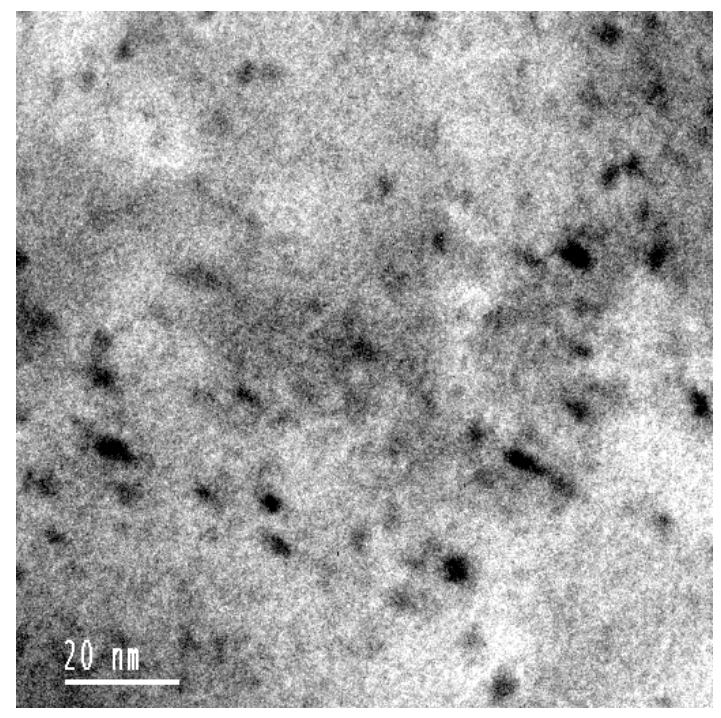

Figure 7. Near $<111>\{220\}$ bright field diffraction contrast image of $\mathrm{Pb}(\mathrm{Se}, \mathrm{Te})$. There are no "blackwhite" and "coffee-bean" contrasts which would identify strain fields. The visibility of approximately $5 \mathrm{~nm}$ wide entities that probably arises from absorption contrast suggests significant differences in the chemical composition.

Since there are no visible strain fields, our hypothesis is that these entities are some kind of (atomically ordered) $\mathrm{Pb}-\mathrm{Se}-\mathrm{Te}$ compound precipitates with lattice constants and respective orientation relationships that are elastic mismatch energy minimizing with respect to the surrounding halite structure $\mathrm{Pb}(\mathrm{Se}, \mathrm{Te})$ matrix. It is not clear if these entities are embedded in the wetting layer, which would require that they are quasi-two dimensional, or if they are sitting on top of the wetting layer. These entities may, anyhow, be considered as predecessors of atomically ordered quantum dots in this materials system.

Consistent with our hypothesis on atomic ordering, the selected area electron diffraction pattern of the same area showed a variety of superlattice reflections, Fig. 8, that cannot arise from a crystal with the halite structure. Corresponding superlattice reflections were also observed in Fourier transform power spectra of [111] zone-axis high-resolution 
phase-contrast images from the same specimen region (to be shown elsewhere). [112] zone-axis selected area electron diffraction pattern (to be shown elsewhere) from the same specimen region, also showed superlattice spots and streaks perpendicular to the transmitted electron beam direction.

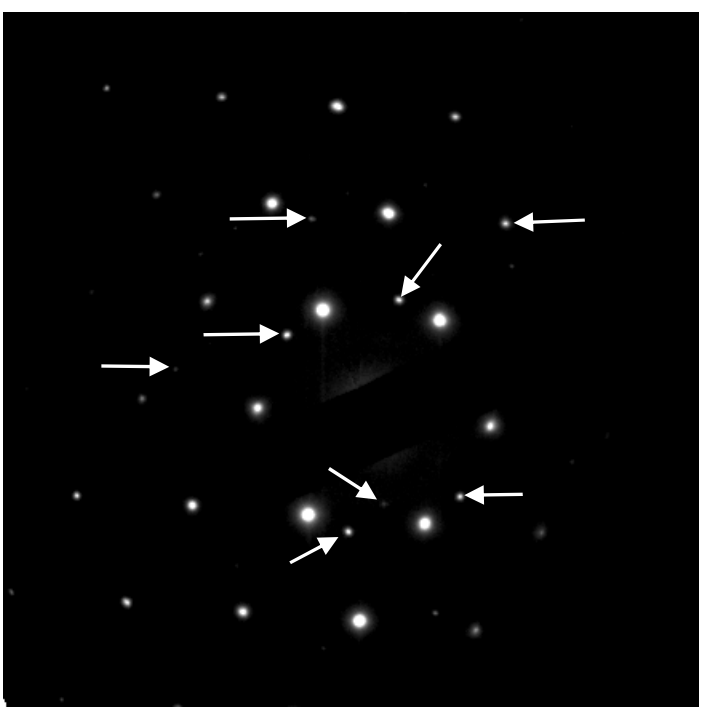

Figure 8. $<111>$ zone-axis selected area electron diffraction pattern showing a variety of superlattice reflections that we assume to be due to $\mathrm{Pb}-\mathrm{Se}-\mathrm{Te}$ compounds. A few of these superlattice spots (which are forbidden in the halite structure) are marked by arrows. (The faint vertical lines in this diffraction pattern are artifacts of the charge-coupled-device camera used for the recording of this image.)

\section{Summary and Conclusions}

The coexistence of smaller and larger ordinarily strained $\mathrm{Pb}(\mathrm{Se}, \mathrm{Te})$ islands, i.e. ordinarily strained quantum dot predecessor structures, was observed by both AFM and TEM. The dominate variety of nanoislands possesses an average height of $16.9 \mathrm{~nm}$ with a standard deviation of $4.6 \mathrm{~nm}$ and a relative spread of $27.2 \%$. The number density of these nano-islands is $41 \mu^{-2}$.

Regions with small atomically ordered entities that consist probably of (atomically ordered) $\mathrm{Pb}-\mathrm{Se}$ Te compounds were also identified in the same specimen. These islands may be considered as constituting predecessors of atomically ordered quantum dots.

In order to reveal the shape of the nano-island, AFM studies with sharper tips and in the contract mode will be performed in the near future.

\section{Acknowledgments}

This research was supported by an award from Research Corporation. Additional support from the Portland State University Foundation is acknowledged. The TEM investigations were supported by the Campus Research Board of the University of Illinois at Chicago and the National Science Foundation (Grant DMR-9733895 to NDB).

\section{References}

[1] D. Bimberg, "Quantum dots: Paradigm changes in semiconductor physics", Semiconductors 33, 951 (1999).

[2] D. Bimberg, M. Grundmann and N.N. Ledentsov, Quantum Dot Heterostructures, (Wiley, 1999).

[3] T.P. Pearsall (editor), Quantum Semiconductor Devices and Technologies, (Kluwer, 2000).

[4] G. Springholz, T. Schwarzl, W. Heiss, G. Bauer, M. Aigle, H. Pascher, and I. Vavra, "Midinfrared surface-emitting $\mathrm{PbSe} / \mathrm{PbEuTe}$ quantum-dot lasers" Appl. Phys. Lett. 79, 1225 (2001).

[5] P. Möck, T. Topuria, N. D. Browning, M. Dobrowolska, S Lee, J. K. Furdyna, G. R. Booker, N. J. Mason, and R. J. Nicholas, "Internal self-ordering in $\mathrm{In}(\mathrm{Sb}, \mathrm{As}),(\mathrm{In}, \mathrm{Ga}) \mathrm{Sb}$, and (Cd,Zn,Mn)Se nano-agglomerates/quantum dots", Appl. Phys. Lett. 79, 946 (2001).

[6] P. Moeck, "Quantum Dots, Semiconductor: Atomic Ordering Over Time", in: Encyclopedia of Nanoscience and Nanotechnology, (Marcel Dekker, 2004).

[7] A. Zunger and S. Mahajan, "Atomic ordering and phase separation in epitaxial III-V alloys", in Handbook on Semiconductors (Elsevier,1994).

[8] A. Mascarenhas (editor), Spontaneous Ordering in Semiconductor Alloys, (Kluwer, 2002).

[9] MRS Bulletin, issue on "Compositional Modulation and Ordering in Semiconductors", Vol. 22, No. 7, 1997, guest editors: T.P. Pearsall and G.B. Stringfellow.

[10] L. Salamanca-Young, D.L. Partin, and J. Heremans, "Ordering and stability of $\mathrm{Pb}_{1-\mathrm{x}} \mathrm{Eu}_{\mathrm{x}}$ Te", J. Appl. Phys. 63, 1504 (1988)

[11] A. Krost, B. Harbecke, R. Faymonville, H. Schlegel, E.J. Fantner, K.E. Ambrosch, and G. Bauer, J. Phys. C 18, 2119 (1985).

[12] P. Möck, K. Pierz, T. Topuria, N.D. Browning, H. Wu, and P.J. McCann, "Atomic ordering in Self-assembled Epitaxial II-VI and IV-VI Compound Semiconductor Quantum Dot Systems", Mat. Res. Soc. Symp. Proc. 749, W13.5.1 (2003).

[13] A.G. Cullis, D.J. Norris, T. Walter, M.A. Migliorato, and M. Hopkinson, "Epitaxial island growth and the Stranski-Krastanow Transition", Phys. Rev. B 66, 081305-1 (2002).

[14] P. Möck, G.R. Booker, N.J. Mason, R.J. Nicholas, E. Alphandéry, T. Topuria, N.D. Browning, "MOVPE grown selfassembled and self-ordered $\mathrm{InSb}$ quantum dots in a GaSb matrix assessed by means of AFM, CTEM, HRTEM and PL", Mater. Sci. Engin. B 80112 (2001)

[15] J.G. Griffith and D.A. Grigg, "Dimensional metrology with scanning probe microscopes", J. Appl. Phys. 74, R83 (1993).

[16] M. Pinczolits, G. Springholz, and G. Bauer, "Molecular beam epitaxy of highly faceted self-assembled IV-VI quantum dots with bimodal size distribution", J. Cryst. Growth 201-202, 1126 (1999)

[17] M. Pinczolits, G. Springholz, and G. Bauer, "Direct formation of self-assembled quantum dots under tensile strain by heteroepitaxy of PbSe on PbTe (111)", Appl. Phys. Lett. 73, 250 (1998). 